\title{
Stability analysis by a nonlinear upper bound on the derivative of Lyapunov function
}

\author{
Gökhan Şahan \\ Department of Mathematics, Izmir Institute of Technology, Urla, Izmir, Turkey
}

\section{A R T I C L E I N}

\section{Article history:}

Received 16 October 2019

Revised 24 January 2020

Accepted 19 February 2020

Available online 10 March 2020

Recommended by Prof. T Parisini

\section{Keywords:}

Asymptotic stability

Nonlinear systems

Lyapunov method

Indefinite Lyapunov function

Bellman-Gronwall inequality

Perturbation of linear time varying systems

\begin{abstract}
A B S T R A C T
In this work, we give results for asymptotic stability of nonlinear time varying systems using Lyapunovlike Functions with indefinite derivative. We put a nonlinear upper bound for the derivation of the Lyapunov Function and relate the asymptotic stability conditions with the coefficients of the terms of this bound. We also present a useful expression for a commonly used integral and this connects the stability problem and Lyapunov Method with the convergency of a series generated by coefficients of upper bound. This generalizes many works in the literature. Numerical examples demonstrate the efficiency of the given approach.
\end{abstract}

(C) 2020 European Control Association. Published by Elsevier Ltd. All rights reserved.

\section{Introduction}

Relaxation of the Lyapunov's Direct Method is recently one of the most common problems for engineering studies. Even though Lyapunov Function (LF) is required to have a decreasing fashion, recent studies have shown that stability can be proved with a LF which has an indefinite derivative as well. However, the amount of the interval that LF can increase or the magnitude of the LF for these intervals and how it is related with the corresponding system structure are quite difficult problems for various types of nonlinear systems, Ahmadi [2] and Michel et al. [21].

One of the research directions for the relaxation of the LF $V$ is to put an upper bound function for $\dot{V}$ instead of 0 . The pioneer work of Michel [20] proposed to put an upper bound $G(V, t)$ and so gave some conditions for the stability of nonlinear time varying systems. A similar idea has also been applied to some different systems, Grujic [9]. Structure of $G(V, t)$ has been specified in the forms $\phi(t)$ or $u(t) V^{m}(x(t))$ for practical and finite time stability (PS, FTS) of the system by Michel and Porter [22] and Chen and Yang [5]. Details about PS and its relation with LF can be found in the book of Laksmikhantam et al. [14]. $n=1$ case of the latter form was also used by $\mathrm{Wu}$ and Sun [30] but for the stability of stochastic systems, introducing the concept of p-moment stability.

\footnotetext{
E-mail addresses: gsahan@iyte.edu.tr, sahangokhan@yahoo.com
}

Some other following works improved this idea for stochastic systems with Markovian Switching as well, Peng and Zhang [27].

The idea to bound $\dot{V}$ by a linear form of $V$, i.e. to put the condition

$\dot{V}(t, x) \leq \mu(t) V(t, x)$

and to give conclusions for asymptotic, exponential, uniform and input to state stability (AS, ES, US and ISS) for general nonlinear systems or nonlinear delay systems was also utilized recently by numerous works. However, many authors added some conservative conditions on the coefficient $\mu(t)$ such as finiteness [4,25,27,31] or being bounded by another function $[18,19]$. However this assumption has been removed by some works of Chen and Yang [3], Zhou [33] and Zhou and Zhao [35]. This new idea also has been the subject of Linear Time-Varying (LTV) Systems $[3,32]$ and Time-Varying Switched Systems (TVSS) [4,10] for stability and stabilizability of the system. For all of these works, the linear bound condition (1) has been given with the condition

$\alpha_{1}(\|x\|) \leq V(t, x) \leq \alpha_{2}(\|x\|)$

where $\alpha_{i}$ belongs to class $K, K_{\infty}$ or some other comparison function classes, $i=1,2 . \alpha_{i}$ 's have been adapted as matrix bounds for LTV systems by Chen and Yang [3] and Zhou [32] because of the structure of $V(t, x)$ in LTV systems and converted to some other different forms for TVSS by Junjie et al. [10]. The conclusions drawn in these works generalized many results in the existing literature. 
The structure of (1) has been improved by some subsequent works. For the AS of an uncontrolled system

$\dot{x}=f(t, x), \quad x\left(t_{0}\right)=x_{0}, \quad t_{0} \geq 0$

where $f \in C\left[J \times \mathbb{R}^{n}, \mathbb{R}^{n}\right]$, the forms

$\dot{V}(t, x) \leq g(t) \alpha(V(t, x)), \dot{V}(t, x) \leq-\alpha(V(t, x))$

were used by Chen and Yang [3] and Lin, Sontag and Wang [17], and

$\dot{V}(t, x) \leq \mu(t) V(t, x)+\pi(t)$

was used by Mazenc and Malisof [18] and Zhou [33] for the function $V \in C^{1}\left[J \times \mathbb{R}^{n}, \mathbb{R}\right]$ with some additional assumptions on $\alpha(V(t$, $x)$ ), $g(t), \mu(t)$ and $\pi(t)$. On the other hand, for a controlled system $\dot{x}=f(x, d)$, the forms

$\dot{V}(t, x) \leq-\alpha(|x|)+\sigma(|d|)$

$\dot{V}(x) \leq-\alpha(|x|)+g(|x|,|d|)$

were utilized by Sontag and Wang [29] and Liberzon and Shim [16] respectively, again with some additional assumptions on $\alpha, \sigma$ and $g$. Differing from the others, Liberzon and Shim [16] also relates ISS with the limit of the ratio

$\frac{g(|x|,|d|)}{\alpha(|x|)}$.

There exist also some works that put some similar upper bounds on $\dot{V}(t, x)$ for small gain theorem on ISS networks, for AS of a controlled time-varying system, for ISS of nonlinear delay systems or to construct Lyapunov-Krasovskii Functionals, (see, [6,12,26,34], respectively).

In this study, we improve the conditions above and give results for AS of system (2) using the form

$\dot{V}(t, x) \leq \pi(t) V^{m}(t, x)+\mu(t) V(t, x)$

where $1 \neq m \in \mathbb{R}$. In addition, we alternatively change or remove the conditions given together with (4) by Mazenc and Malisof [18] and Zhou [33] and recommend some other conditions using the integral expressions in Lemma 2 below. This converts the stability problem into a convergency and limit problem of an infinite series, and also relates the stability problem with the ratio of the coefficients in (7) for some cases, thus connecting the problem with the existing literature [16].

Nomenclature: Throughout the paper we use the following abbreviations and definitions. By the negative powers of $V$, we mean the multiplicative inverse of it, not functional inverse; $\mathbb{R}$ is the set of real numbers; $J:=[0, \infty), J^{+}:=(0, \infty), J^{-}:=(-\infty, 0)$; by $C^{n}[A, B]$ we mean $n$ times differentiable functions from $A$ to $B ; P C$ represents piecewise continuous functions, and $K$ and $K_{\infty}$ are the family of class $K$ and $K_{\infty}$ functions [13].

\section{Stability of nonlinear time-varying systems}

Throughout this work we will consider the uncontrolled nonlinear time-varying system (2). We assume that the vector field $f(t$, $x$ ) is piecewise continuous in $t$ and locally Lipschitz in $x$.

Definition 1. [13] The equilibrium $x=0$ of (2) is said to be:

- stable if, for each $\epsilon>0$, there is a $\delta\left(\epsilon, t_{0}\right)>0$ such that

$\left\|x\left(t_{0}\right)\right\|<\delta \Rightarrow\|x(t)\|<\epsilon, \forall t \geq t_{0} \geq 0 ;$

- uniformly stable if, for each $\epsilon>0$, there is a $\delta(\epsilon)>0$, independent of $t_{0}$, such that (8) is satisfied;

- AS if it is stable and there is a positive constant $c=c\left(t_{0}\right)>0$ such that $x(t) \rightarrow 0$ as $t \rightarrow \infty$, for all $\left\|x\left(t_{0}\right)\right\|<c$.
We give the concept of stable function which was introduced in [32].

Lemma 1. Consider the following scalar LTV system

$\dot{y}(t)=\mu(t) y(t), \quad t \in J$

where $y(t): J \rightarrow \mathbb{R}$ is the state function, $\mu(t) \in P C(J, \mathbb{R})$. Then $\mu(t)$ is AS if the system (9) is AS which is equivalent to

$\lim _{t \rightarrow \infty} \int_{t_{0}}^{t} \mu(\lambda) d \lambda=-\infty$

Now, we state a motivating example which explains the idea of our main results.

Example 1. Consider the following system.

$\dot{x}=t \sin (t) x-\frac{t^{3} x^{3}}{2}, \quad x\left(t_{0}\right) \neq 0, \quad t \in(1, \infty)$

and the function $V(x)=x^{2}$. Then,

$$
\begin{aligned}
\dot{V}(x) & =2 t \sin (t) x^{2}-t^{3} x^{4} \\
& \leq 2 t V(x)-t^{3} V^{2}(x) \\
& =: \mu(t) V(x)+\pi(t) V^{2}(x)
\end{aligned}
$$

Note that the right hand side of this inequality is not strictly negative and thus the classical Lyapunov approach cannot be applied. Also the recently developed methods in works $[3,4,18,25,33]$ that assume the right hand side of the above inequality as $\mu(t) V(x)$ or $\mu(t) V(x)+\pi(t)$ such that $\mu(t)$ is AS cannot be performed. This is because of both the instability of $\mu(t)$ and the factor $V^{m}$ of $\pi(t)$.

On the other hand, to deal not only with the first power of the candidate Lyapunov Function but also with some different powers of $V$ is quite a common case in the literature. Higher or lower order powers of $V$ are encountered in sliding mode control (see p.2859 of [23]), in the construction of Lyapunov Krasovskii functional for time-varying systems with delay, (see Assumption 4 of the work [18]) and in some other works.

Therefore, a more general structure on the upper bound of $\dot{V}(t, x)$ makes the nonlinear system analysis easier and the Lyapunov Method more flexible, providing more suitable LF's to utilize in analysis.

Now we continue to solve the problem above and to explain the method that we'll use. We multiply the each side of $(8)$ by $-V^{-2} e^{t^{2}}$ and revise. Then, we have

$\frac{d}{d t}\left[V^{-1} e^{t^{2}}\right] \geq t^{3} e^{t^{2}}$

$\Rightarrow V(x) \leq \frac{e^{t^{2}}}{\int_{t_{0}}^{t} \tau^{3} e^{\tau^{2}} d \tau+V_{0}}$

where $V_{0}:=\frac{e^{t^{2}}}{x_{0}^{2}}$. The integral of the type $\int_{t_{0}}^{t} f(\tau) e^{g(\tau)} d \tau$ is quite a common integral for many applications. Thus, it has been studied by many authors (see the argument in p.1437 of [33] and the references therein). While some classes of functions were developed to bound the exponential part of integrand by Kalman [11], some other conclusions were also given for this integral to be bounded and its limit to be zero by Gelig et al. [8]. For the AS of the system (2), at this stage, the work [33] proposes to assume this class of integrals to be bounded and its limit to be zero.

We alternatively will recommend some other conditions instead of these. According to the structure of $\pi(t)$ and $\mu(t)$, this condition relates the problem with the ratio of the functions $f(\tau)$ and $g(\tau)$. (Here note that $f(\tau)$ is actually $(1-m) \pi(\tau)$ and $g(\tau)$ is $(m-1) \int \mu(\lambda) d \lambda$ that lie in the upper bound) 
Consider the following integral

$\int \tau^{3} e^{\tau^{2}} d \tau$

It can be solved as

$\int \frac{d}{d \tau}\left[\left(\frac{\tau^{2}}{2}-\frac{1}{2}\right) e^{\tau^{2}}\right]$.

With a general overview, we construct the ratio $\frac{f(\tau)}{g^{\prime}(\tau)}$ first and then divide the derivation of this ratio by $-g^{\prime}(\tau)$ again for the next integral, if necessary.

Although we obtain finite terms depending on the structure of the functions $f(\tau)$ and $g(\tau)$, we may have an infinite and divergent series as well. In addition, instead of using the higher order derivatives, it's also possible to use higher order integrals and again integration by parts. So one may also attempt to write integral (12) as follows by performing integration by parts infinite times.

$\int \frac{d}{d \tau}\left[\left(\frac{\tau^{4}}{4}+\frac{\tau^{6}}{4.6}(-2)+\cdots+\frac{\tau^{2 n+2}}{4.6 \ldots(2 n+2)}(-2)^{n-1}+\cdots\right) e^{\tau^{2}}\right]$

Here note that while one of the terms of $\frac{d}{d \tau}\left(\frac{\tau^{4}}{4} e^{\tau^{2}}\right)$ is giving the integrand of (12), the other term remains as a surplus term. But the next resulting term cancels it out and the process follows this path. However, mathematically, this is only possible when the series is convergent, Agarwal et al. [1]. Notice that the series

$\frac{\tau^{4}}{4}+\frac{\tau^{6}}{4.6}(-2)+\cdots+\frac{\tau^{2 n+2}}{4.6 \ldots(2 n+2)}(-2)^{n-1}+\cdots$

is a convergent power series for any finite $\tau$ values. This time, the methodology to construct each term is based on integration of $f(\tau)$ first, say $h_{0}$, and then integration of $g(\tau) h_{0}$.

We conclude these conditions formally as follows.

Lemma 2. Consider the integral

$\int f(t) e^{g(t)} d t$

where $f, g \in C(J, \mathbb{R})$ and the following series':

1. $H_{1}(t)=h_{0}(t)+h_{1}(t)+\cdots$ such that

$h_{0}(t)=\int f(t) d t, \quad h_{j+1}(t)=\int h_{j}(t)\left(-g^{\prime}(t)\right) d t$,

$j=0,1,2, \ldots$

2. $H_{2}(t)=h_{0}(t)+h_{1}(t)+\cdots$ such that $g^{\prime}(t) \neq 0$ for $t \in J$ and

$$
h_{0}(t)=\frac{f(t)}{g^{\prime}(t)}, \quad h_{j}(t)=\frac{\frac{d}{d t}\left(h_{j-1}(t)\right)}{-g^{\prime}(t)}, \quad j=0,1,2, \ldots
$$

If $H_{i}(t)$ is convergent for $i=1$ or 2 , then

$\int f(t) e^{g(t)} d t=\int \frac{d}{d t}\left[H_{i}(t) e^{g(t)}\right]=H_{i}(t) e^{g(t)}$.

Proof. The ith terms are cancelled out by the previous or later terms for $i>0$. Thus the proof can be done easily using mathematical induction.

Now, let us turn back to Example 1. As a result, (11) can be written as

$V(x) \leq \frac{1}{\frac{t^{2}-1}{2}+K_{0} e^{-t^{2}}}, K_{0} \in \mathbb{R}$.

The righthand side of (16) is bounded and its limit tends to zero for the given region. Thus the system that we consider in Example 1 is asymptotically stable.

Consequently, we both used the different powers of the Lyapunov Function and related the problem with the convergency and behaviour of the resulting finite/infinite series. These terms are developed from the relation of $m, \pi$ and $\mu$ as well.

Here, to obtain the AS of the system in Example 1, we needed the boundedness and zero limit. These two conditions can also be received automatically in case of taking the functions $f(t)$ and $g(t)$ from some special classes, [8] and Lemma 3 of [33]. However, the zero limit condition itself can also imply the boundedness for some $f$ and $g$ as the following lemma shows.

Lemma 3. Consider the function

$\kappa\left(t, t_{0}\right):=\int_{t_{0}}^{t} f(\tau) e^{g(\tau)} d \tau$

where $f \in P C(J, J), g \in C(J, \mathbb{R})$.

1. If $g(t) \rightarrow-\infty$ as $t \rightarrow \infty$ and $\lim _{t \rightarrow \infty} \kappa\left(t, t_{0}\right)=0$, then the function $\kappa\left(t, t_{0}\right)$ is bounded.

2. If $\lim _{t \rightarrow \infty} \kappa\left(t, t_{0}\right)=\infty$, then the function $\kappa\left(t, t_{0}\right)$ is bounded from below.

Now we generalize the idea in Example 1.

Theorem 4. Consider (2) and assume that there exist

$\cdot V: J \times \mathbb{R}^{n} \rightarrow J, V \in \mathcal{C}^{1}$;

- $\alpha \in \mathcal{K}$;

- $\mu(t) \in \mathcal{C}^{\infty}(J, \mathbb{R})$;

- $\pi(t) \in \mathcal{C}^{\infty}(J, I), I= \begin{cases}J^{+}, & m<1 \\ J^{-}, & m>1\end{cases}$

such that

$\alpha(\|x\|)<V(t, x)$,

and (7) holds. Assume also that one of the series $H_{i}(t): J \rightarrow$ $\mathbb{R}$ in Lemma 2 is convergent for $f(t):=\pi(t)$ and $g(t):=(m-$ 1) $\int_{t_{0}}^{t} \mu(\lambda) d \lambda:=\bar{\mu}(t)$ provided that

$\lim _{t \rightarrow \infty} H_{i}(t)=L$

exists or $\pm \infty$. Then system (2) is AS if one of the following conditions hold.

1. $m<1, \mu(t)$ is $A S$ and $L=0$,

2. $m>1$ and $\mu(t)$ is $A S$,

3. $m>1$ and $L=-\infty$.

Proof. 1) Let $m<1$. Multiply each side of (7) by (1$m) V^{-m}(t, x) \exp (\bar{\mu}(t))$ and revise. Then we have the following for $t \geq t_{0}$ :

$\frac{d}{d t}\left[V^{1-m}(t, x) \exp (\bar{\mu}(t))\right] \leq(1-m) \pi(t) \exp (\bar{\mu}(t))$

$V^{1-m}(t, x) \exp (\bar{\mu}(t))-V_{0} \leq \int_{t_{0}}^{t}(1-m) \pi(\tau) \exp (\bar{\mu}(\tau)) d \tau$

where $V_{0}:=V^{1-m}\left(t_{0}, x_{0}\right)$. Now, by Lemma 2, (19) can be written as follows,

$$
\begin{aligned}
& V^{1-m}(t, x) \exp (\bar{\mu}(t)) \leq(1-m) \int_{t_{0}}^{t} \frac{d}{d \tau}\left[H_{i}(\tau) \exp (\bar{\mu}(\tau)]+V_{0}\right. \\
& =(1-m) H_{i}(t) \exp (\bar{\mu}(t))+K_{0}
\end{aligned}
$$

such that

$K_{0}:=V_{0}-(1-m) H_{i}\left(t_{0}\right)$.

Note that we ensure the positivity of the righthand side of (19) because of the definition of $\pi$. Using also inequality (17), we have

$\alpha(\|x(t)\|) \leq V(t, x(t)) \leq\left[(1-m) H_{i}(t)+\frac{K_{0}}{\exp (\bar{\mu}(t))}\right] \frac{1}{1-m}$. 
Define the function

$G\left(t, x_{0}\right):=(1-m) H_{i}(t)+\frac{K_{0}}{\exp (\bar{\mu}(t))}$.

We know by Lemma 3-1 that the right hand side of (19) is bounded and so $(1-m) H_{i}(t)$ is. For any arbitrarily choosen $\epsilon>0$, there exists such a $\delta\left(\epsilon, t_{0}\right)>0$ that

$$
\begin{aligned}
\alpha(\|x(t)\|) & \leq V(t, x(t)) \leq\left[G\left(t, x_{0}\right)\right] \frac{1}{1-m} \\
& \leq\left[\bar{G}\left(t_{0}, x_{0}\right)\right] \frac{1}{1-m} \leq \alpha(\epsilon)
\end{aligned}
$$

where $\bar{G}\left(t_{0}, x_{0}\right):=\sup _{t \geq t_{0}} G(t, x)$ for $\left\|x_{0}\right\|<\delta$. This implies $\|x(t)\| \leq \epsilon$ for $t \geq t_{0}$.

The asymptotic convergence is also implied as $V(t, x) \rightarrow 0$ as $t \rightarrow \infty$.

2-3) Now assume that $m>1$. This time we have

$$
\begin{gathered}
V^{1-m}(t, x) \exp (\bar{\mu}(t)) \geq \int_{t_{0}}^{t}(1-m) \pi(\tau) \exp (\bar{\mu}(\tau)) d \tau+V_{0} \\
\Rightarrow V^{1-m}(t, x) \geq(1-m) H_{i}(t)+\frac{K_{0}}{\exp (\bar{\mu}(t))}
\end{gathered}
$$

We guaranteed the positivity of the righthand side of (23) and so (24) by conditions of the theorem. This also shows that $H_{i}(t)<0$, $K_{0}>0$. As a result, it's possible to get a similar form with (21) as follows.

$\alpha(\|x(t)\|) \leq V(t, x(t)) \leq\left[\frac{1}{(1-m) H_{i}(t)+\frac{K_{0}}{\exp (\bar{\mu}(t))}}\right] \frac{1}{m-1}$ have

Now define $\bar{E}\left(t_{0}\right):=\sup _{t \geq t_{0}} \exp (\bar{\mu}(t))$. Then for any $\epsilon>0$, we $\alpha(\|x(t)\|) \leq V(t, x(t))$

$\leq\left[\frac{1}{G\left(t, x_{0}\right)}\right]^{\frac{1}{m-1}} \leq\left[\frac{\bar{E}\left(t_{0}\right)}{K_{0}}\right]^{\frac{1}{m-1}} \leq \alpha(\epsilon)$

for $\left\|x_{0}\right\|<\delta$. This implies $\|x(t)\| \leq \epsilon, t \leq t_{0}$. for Case 2 ).

This time we define $\underline{G}\left(t_{0}, x_{0}\right):=\operatorname{Inf} f_{t \geq t_{0}} G(t, x)$ with the help of Lemma 3-2. So (26) should be written as follows.

$\alpha(\|x(t)\|) \leq V(t, x(t))$

$\leq\left[\frac{1}{G\left(t, x_{0}\right)}\right]^{\frac{1}{m-1}} \leq\left[\frac{1}{\underline{G}\left(t_{0}, x_{0}\right)}\right]^{\frac{1}{m-1}} \leq \alpha(\epsilon)$

for $\left\|x_{0}\right\|<\delta$. This implies $\|x(t)\| \leq \epsilon, t \leq t_{0}$.

For the cases 2 to 3 , asymptotic convergence is also implied as the denominator part tends to $\infty$ when $\mu(t)$ is AS or $L=-\infty$. But note that for all of the items, it's not possible to remove the dependency on $t_{0}$. Thus we can not get uniform stability.

Remark 1. (Comparison with existing literature)

As well as relaxing the monotonicity constraint of classical LF, Theorem 4 of our work also improves the conditions of $[3,18,33]$ from many aspects.

The works $[18,33]$ present stability conditions by putting a linear upper bound function as (4). While [18] puts some additional assumptions on $\mu(t)$ as boundedness, in the work [33]

- Theorem 1 utilizes the AS of $\mu(t)$ in case of $\pi(t)=0$.

- Theorem 2 utilizes the AS of $\mu(t)$, boundedness of $\kappa(t)$ and $L=0$ condition for $m=0$ where

$$
\kappa(t):=\int_{t_{0}}^{t} \pi(\tau) \exp (\mu(\tau)) d \tau, \lim _{t \rightarrow \infty} \kappa(t)=L
$$

We showed that the boundedness assumption of $\kappa\left(t, t_{0}\right)$ in Theorem 2 of [33] is already implied by the rest of the hypothesis. In addition, we generalized Theorem 2 of [33]. Consider

$\bar{\kappa}(t):=(1-m) \int_{t_{0}}^{t} \pi(\tau) \exp (\bar{\mu}(\tau)) d \tau$.

We replaced the conditions of having a bounded $\kappa(t)$ and $L=0$ with just the $L=0$ condition of (18). It is also equivalent to say that $\lim _{t \rightarrow \infty} \bar{\kappa}(t)=0$. However, we also gave the AS of (2) for any $m \in \mathbb{R}$ in (7). We even removed the AS of $\mu(t)$ for some $m$ values. When $\pi(t)$ and $\mu(t)$ are some suitable polynomials, it's easier to verify the AS of (2).

On the other hand, Lemma 1 and 2 of [3] also use the AS of $\mu(t)$ and $\pi(t)=0$ form of (7). Hence, it's a special case of Theorem 4 of our work.

The works [3,32] adapt these conditions to Linear Time Varying systems and generalize numerous works in the literature. AS

From the point of view of our Theorem 4, briefly [3,32] present

- Assuming that the vector field $f$ in $(2)$ is linear, $\pi(t)$ in (7) is zero,

- Changing the function $\alpha$ in (17) with matrix bounds.

There are also many works which are generated by the conclusions of works [3,32].

Now, let us interpret Example 1 from the point of view of Theorem 4 above. In Example 1,

- $m=2$,

- $\mu(t)=2 t$ and not AS,

- $H_{i}(t)$ should be choosen as $\frac{1-t^{2}}{2}$. It is the $i=2$ case for Lemma 2, so $\mathrm{H}_{2}(t)$.

$\mathrm{H}_{2}(t)$ tends to $-\infty$ as $t \rightarrow \infty$, and so holds conditions of Theorem $4-3$, and thus the system is AS.

We give some more examples that explain the use of the rest of the items in Theorem 4 and that show the efficiency of the given conditions.

Example 2. Consider the following system

$\dot{x}=\frac{-3 t^{2}}{4} x+t \frac{x}{x^{4}+1}, \quad t \in J$,

and the candidate Lyapunov Function $V(x)=x^{2}$. Then,

$$
\begin{aligned}
\dot{V}(x) & =\frac{-3 t^{2}}{2} x^{2}+2 t \frac{x^{2}}{x^{4}+1} \\
& \leq \frac{-3 t^{2}}{2} x^{2}+2 t x^{-2} \\
& =: \mu(t) V(x)+\pi(t) V^{-1}(x)
\end{aligned}
$$

Now we assess the example in view of Theorem 4 .

- $m=-1$,

- $\mu(t)$ is AS,

- $H_{2}(t)=\frac{1}{3 t}+\frac{1}{3^{2} t^{4}}+\frac{4}{3^{3} t^{7}}+\cdots \frac{3 n-2}{3^{n+1} t^{3 n+1}}+\cdots$,

- $L=0$

The series $H_{2}(t)$ is convergent for $t>t_{0}^{*}$, and this constant $t_{0}^{*}$ is determined by the coefficients of $\mu(t)$ and $\pi(t)$. The system is AS by Theorem $4-1$. We actually get this conclusion without solving the system and without using a classical LF.

Remark 2. Observation of Example 1-2 yields that our methodology is especially effective for some degenerated forms of Bernoulli Differential Equation, Murphy [24] and Ross [28]. Here, the crucial point is to enlarge $\dot{V}(t, x)$ by performing some algebraic operations and finally to achieve a Lyapunov inequality form of the 
Bernoulli Differential Equation and appropriate $\mu(t)$ and $\pi(t)$ coefficients for hypothesis of Theorem 4 . Then conclusions can be given using Theorem 4 above.

Example 3. Consider the following system which is a perturbed version of Liao et al. [15]-Example 4.6.3, Zhou [33]-Example 1, Lakshmikantham et al. [13]-Example 4.18.

$\dot{x}=-\frac{x}{t+\sin (x)}+p(t) x^{2 m-1}, \quad t \in J=(1, \infty)$,

where $1 \neq m \in \mathbb{R}, \quad p(t)$ is a rational polynomial for $t \in J$. Consider also a function $V(x)=x^{2}$. Then,

$$
\begin{aligned}
\dot{V}(x) & =-\frac{2 x^{2}}{t+\sin (x)}+2 p(t) x^{2 m}(x) \\
& \leq-\frac{2}{t+1} V(x)+2 p(t) V^{m}(x) \\
& =\mu(t) V(x)+\pi(t) V^{m}(x)
\end{aligned}
$$

Here, $\mu(t)=\frac{-2}{t+1}$ is an AS function. Bearing in mind the conditions of Theorem 4, it's possible to give the amount of perturbation by $p(t)$.

Let $m<1, p(t)>0$ for $t \in J$. The system (29) is AS for any choice of $p(t)$ provided that $H_{i}(t)$ is convergent and $L=0$. For example, if $i=2$, then we only check the convergency and $L=0$ case for the following series

$\frac{p(t)(t+1)}{1-m}+\frac{\frac{d(p(t)(t+1)}{d t}(t+1)}{-2(1-m)^{2}}+\ldots$

and it's computationally tractable.

Now let us give a multidimensional example that demonstrates the quality of the conclusions of our main result.

Example 4. Consider the following system.

$\left[\begin{array}{c}\dot{x}_{1} \\ \dot{x}_{2}\end{array}\right]=\left[\begin{array}{c}\mu_{1}(t) x_{1}+\mu_{2}(t) x_{2}+\pi_{1}(t) x_{1}^{3}+\pi_{2}(t) x_{1} x_{2}^{2} \\ -\mu_{2}(t) x_{1}+\mu_{3}(t) x_{2}+\pi_{3}(t) x_{2}^{3}+\pi_{4}(t) x_{1}^{2} x_{2}\end{array}\right]$

and the function $V=\frac{x_{1}^{2}+x_{2}^{2}}{2}$ where $\mu_{i}(t) \in C^{\infty}(J, \mathbb{R}), \pi_{j}(t) \in$ $C^{\infty}\left(J, J^{-}\right), i=1,2,3 ; j=1,2,3,4$. Then we have

$\dot{V}=\mu_{1}(t) x_{1}^{2}+\mu_{3}(t) x_{2}^{2}+\pi_{1}(t) x_{1}^{4}+\left[\pi_{2}(t)+\pi_{4}(t)\right] x_{1}^{2} x_{2}^{2}+\pi_{3}(t) x_{2}^{4}$.

Let $\mu_{i}$ 's and $\pi_{i}$ 's be upper bounded as follows.

$$
\begin{gathered}
\mu_{i}(t) \leq \mu(t) \text { for } i=1,3 \\
\pi_{j}(t) \leq \pi(t) \text { for } j=1,4 \\
\pi_{k}(t) \leq 2 \pi(t) \text { for } k=2,3 .
\end{gathered}
$$

where $\mu(t) \in C^{\infty}(R, R), \pi(t) \in C^{\infty}\left(R, J^{-}\right)$

$\Rightarrow \dot{V} \leq 2 \mu(t) V+4 \pi(t) V^{2}$

Now we can apply the items 2 and 3 of our main result Theorem 4 . If we choose the upper bounds $\pi(t)<0$ and $\mu(t)$ in such a way that

- The corresponding $\mu(t)$ is AS and $L$ exists or

- $L=-\infty$

Then (30) is AS. Note that, here the system (30) can be written as

$\left[\begin{array}{l}\dot{x}_{1} \\ \dot{x}_{2}\end{array}\right]=\left[\begin{array}{cc}\mu_{1}(t) & \mu_{2}(t) \\ -\mu_{2}(t) & \mu_{3}(t)\end{array}\right]\left[\begin{array}{l}x_{1} \\ x_{2}\end{array}\right]+\left[\begin{array}{l}\pi_{1}(t) x_{1}^{3}+\pi_{2}(t) x_{1} x_{2}^{2} \\ \pi_{3}(t) x_{2}^{3}+\pi_{4}(t) x_{1}^{2} x_{2}\end{array}\right]$

So the system (30) can be regarded as a perturbed LTV system. Observation of the Example 4 yields that Theorem 4 of this work also give the amount of perturbation for an AS LTV system.

\section{Conclusion and future works}

In summary, this paper addresses the stability problem of nonlinear time-varying systems using LF with indefinite derivative. The Lyapunov inequality version of the classical Bernoulli Differential Equation is utilized, and so numerous works in the literature are generalized. This adaptation can also be regarded as a different version of the Bellman-Gronwall Inequality, Flett [7] and its application. In addition, a useful integral expression is given and so a correlation is obtained between the stability and the convergency of the series generated by ratio of the corresponding coefficients. As a future work, the same idea can be reduced to some similar nonlinear differential equations like Riccati, Euler, [24], [28] and some different upper bounds can be found for $\dot{V}(t, x)$. The same methodology can also be applied for different forms of $G(V, t)$ or for ISS of the nonlinear time-varying systems.

\section{Declaration of Competing Interest}

The authors declare that they have no known competing financial interests or personal relationships that could have appeared to influence the work reported in this paper.

\section{Appendix A.}

\section{Proof. (Lemma 3)}

1. First of all, the functions $f(\tau)$ and $e^{g(\tau)}$ are positive and so $\kappa$ is. As a result, it's bounded from below by 0 . Consider $\left[t_{0}, T\right]$ for a finite $T \in \mathbb{R}$. The function $f(\tau) e^{g(\tau)}$ is a piecewise continuous function by hypothesis and so has finite number of jump discontinuities there, say $t_{0}<t_{1}<t_{2}<\ldots<t_{p}:=T$. Define $I=\{1,2, \ldots p\}$ and

$\kappa\left(t_{i}, t_{0}\right)-\kappa\left(t_{i-1}, t_{0}\right):=\bar{\kappa}_{i}$

where $i \in I$. As $f(\tau) e^{g(\tau)}$ is continuous for the interval $\left(t_{i-1}, t_{i}\right)$ and not infinity at bounds, we guarantee $\bar{\kappa}_{i} \in R$ there.

As the limit of $\kappa$ is zero, we have such a number $a \in \mathbb{R}^{+}$and a moment $T^{*} \geq t_{0}$ by the definition of the limit that $0<\kappa(t$, $\left.t_{0}\right) \leq a$ for $t \geq T^{*}$. Hence it's bounded for $t \in\left[T^{*}, \infty\right)$.

First step is valid for any finite choice of $T$. Choose $T=T^{*}$. As a result, we have $0 \leq \kappa\left(t, t_{0}\right) \leq a+\sum \bar{\kappa}_{i}$.

Consequently, if the functions $f(\tau)$ and $e^{g(\tau)}$ are given piecewise continuous and continuous functions respectively, providing the limit condition, corresponding $\kappa$ function is bounded.

2. Consider $\left[t_{0}, T\right]$ for a finite $T \in \mathbb{R}$, the numbers $t_{i}$ 's and so $I$ and the definition (31) again. Let $M:=\min \left[\bar{K}_{i}\right]$ for $i \in I$. Then $\kappa\left(t, t_{0}\right) \geq p M$. This time as the limit is $\infty$, we have such a number $A \in \mathbb{R}^{+}$and a moment $T^{*}$ by the definition of the limit that $\kappa\left(t, t_{0}\right) \geq A$ for $t \geq T^{+}$. Choosing $T=T^{*}$ we have $\kappa\left(t, t_{0}\right) \geq p M+A$ so bounded from below.

\section{Supplementary material}

Supplementary material associated with this article can be found, in the online version, at doi:10.1016/j.ejcon.2020.02.006.

\section{References}

[1] R.P. Agarwal, C. Flaut, D. ORegan, Power and Taylor series, An Introduction to Real Analysis, CRC Press, 2018.

[2] A.A. Ahmadi, Non-monotonic Lyapunov Functions for Stability of Nonlinear and Switched Systems: Theory and Computation, Ms Thesis, MIT, USA, 2008.

[3] G. Chen, Y. Yang, New stability conditions for a class of linear time varying systems, Automatica 71 (2016) 342-347. 
[4] G. Chen, Y. Yang, Relaxed conditions for the input-to-state stability of switched nonlinear time-varying systems, IEEE Trans. Autom. Control 62 (9) (2017) 4706-4712.

[5] G. Chen, Y. Yang, Finite-time stability of switched nonlinear time-varying systems via indefinite Lyapunov functions, Int. J. Robust Nonlinear Control 28 (5) (2018) 1901-1912.

[6] S. Dashkovskiy, H. Ito, F. Wirth, On a small gain theorem for ISS networks in dissipative Lyapunov form, Eur. J. Control 17 (4) (2011) 357-365.

[7] T.M. Flett, Ordinary differential equations, Differential Analysis, Cambridge University Press, Cambridge, 1980.

[8] A.K. Gelig, G.A. Leonov, V.A. Yakubovich, Stability of nonlinear systems with a nonunique equilibrium state, Izdatel'stvo Nauka, Moscow, 1978. 1st ed

[9] L.T. Grujic, Non-Lyapunov stability analysis of large-scale systems on timevarying sets, Int. J. Control 21 (3) (1975) 401-415.

[10] L. Junjie, S. Zhikun, F. Weijie, S. Shuzhi, Stabilizability of time-varying switched systems based on piecewise continuous scalar functions, IEEE Trans. Autom. Control 64 (6) (2019) 2637-2644.

[11] R.E. Kalman, Contributions to the theory of optimal control, Boletin de la Sociedad Matematica Mexicana 5 (2) (1960) 102-119.

[12] I. Karafyllis, J. Tsinias, A converse Lyapunov theorem for nonuniform in time global asymptotic stability and its application to feedback stabilization, SIAM J. Control Optim. 42 (3) (2003) 936-965.

[13] H. Khalil, Nonlinear Systems, Macmillan Publishing Company, 1992.

[14] V. Lakshmikantham, S. Leela, A.A. Martynyuk, Practical Stability of Nonlinear Systems, World Scientific, Singapore, 1990.

[15] X. Liao, L. Wang, Y. Pei, Stability of Dynamical Systems, Elseiver, 2007.

[16] D. Liberzon, H. Shim, An asymptotic ratio characterization of input-to-state stability, IEEE Trans. Autom. Control 60 (12) (2015) 3401-3404.

[17] Y. Lin, E.D. Sontag, Y. Wang, A smooth converse Lyapunov theorem for robust stability,, SIAM J. Control Optim. 34 (1996) 124-160.

[18] F. Mazenc, M. Malisof, Extensions of Razumikhins theorem and Lyapunov Krasovskii functional constructions for time-varying systems with delay, Automatica 78 (2017) 1-13.

[19] F. Mazenc, M. Malisoff, S.I. Niculescu, Stability analysis for systems with timevarying delay: trajectory based approach, in: Proceedings of the Fifty-Fourth IEEE CDC Osaka,Japan, 2015, pp. 1811-1816.

[20] A.N. Michel, Quantitative analysis of simple and interconnected systems: stability, boundedness, and trajectory behavior, IEEE Trans. Circuit Theory CT-17 (3) (1970).
[21] A.N. Michel, L. Hou, D. Liu, Stability of Dynamical Systems: On the Role of Monotonic and Non-Monotonic Lyapunov Functions, Birkhauser, 2015.

[22] A.N. Michel, D.W. Porter, Practical stability and finite-time stability of discontinuous system, IEEE Trans. Circuit Theory CT-19 (2) (1972) 123-129.

[23] J.A. Moreno, M. Osorio, A Lyapunov approach to second-order sliding mode controllers and observers, in: Proceedings of the Forty-Seventh IEEE CDC, Cancun-Mexico, Dec.9-11, 2008, pp. 2856-2861.

[24] G.M. Murphy, Ordinary Differential Equations and Their Solutions,, Van Nostrand Reinhold Company, 1960.

[25] C. Ning, Y. He, W. Min, Q. Liu, J. She, Input-to-state stability of nonlinear systems based on an indefinite Lyapunov function, Syst. Control Lett. 61 (2012) 1254-1259.

[26] C. Ning, Y. He, M. Wu, S. Zhou, Indefinite derivative Lyapunov Krasovskii functional method for input to state stability of nonlinear systems with time delay, Appl. Math. Comput. 270 (2015) 534-542.

[27] S.G. Peng, Y. Zhang, Some new criteria on Pth moment stability of stochastic functional differential equations with Markovian switching, IEEE Trans. Autom. Control 55 (12) (2010) 2886-2890.

[28] S.L. Ross, 1st order equations for which exact solutions are obtainable, Introduction to Ordinary Differential Equations, Wiley, 4th ed., USA, 1989.

[29] E.D. Sontag, Y. Wang, On characterizations of input-to-state stability with respect to compact sets, in: IFAC Nonlinear Control Systems, 1995, pp. 203-208. California, USA

[30] H. Wu, J. Sun, p-moment stability of stochastic differential equations with impulsive jump and Markovian switching, Automatica 42 (2006) 1753-1759.

[31] M. Zhang, Q. Zhu, Input-to-state stability for non-linear switched stochastic delayed systems with asynchronous switching, IET Control Theory A 13- (3) (2019) 351-359.

[32] B. Zhou, On asymptotic stability of linear time-varying systems, Automatica 68 (2016) 266-276.

[33] B. Zhou, Stability analysis of non-linear time-varying systems by Lyapunov functions with indefinite derivatives, IET Control Theory A 11 (9) (2017) 1434-1442.

[34] B. Zhou, Construction of strict Lyapunov Krasovskii functionals for time-varying time-delay systems, Automatica 107 (2019) 382-397.

[35] B. Zhou, T. Zhao, On asymptotic stability of discrete-time linear time-varying systems, IEEE Trans. Autom. Control 62 (8) (2017) 4274-4281. 